\section{TA-Aktivitäten auf Ebene der Europäischen Union}

\author{
von Michael Rader, ITAS
}

Dieser Beitrag aktualisiert die Übersicht zu TA-Aktivitäten der Einrichtungen der Europäischen Union, die im Rahmen des 1994 in den TA-Datenbank-Nachrichten erschienenen Artikels "Networking in TA" gegeben wurde (Nr. 2, 3. Jahrgang, Juni 1994, S. 914). Es zeigt sich dabei, daß die Rolle der TA im Rahmen der Forschungs- und Technologiepolitik der Kommission zunehmend deutlichere Konturen gewinnt.

\section{Der Beitrag der Europäischen Kommission zur Diffusion von TA}

Ein besonders wichtiger Ausgangspunkt von TA auf europäischer Ebene waren die verschiedenen FAST Programme, und insbesondere das MONITOR/FAST Programm. Ein Ziel dieses Programmes war die Beteiligung von Forschern aus Ländern ohne eigene "TA Tradition" als "Juniorpartner" an europäischen Projekten, an denen Forscher mit TA-Erfahrung prägend mitwirkten. Die Bedeutung solcher Projekte für die Verbreitung des TA-Gedankens, vor allem in den Ländern Südeuropas, wurde immer wieder von Experten aus diesen Ländern, die im Verlauf der Machbarkeitsstudie für ETAI interviewt wurden, hervorgehoben. Die drei vom MONITOR/FAST Programm veranstalteten ECTA (European Congress on Technology Assessment)-Konferenzen spielten in diesem Rahmen eine Schlüsselrolle. Bedauerlicherweise hat die vierte, bereits terminierte, ECTAKonferenz niemals stattgefunden.

Im Gefolge der ECTA-Konferenzen gab es einige von der Europäischen Kommission geförderte Aktivitäten zur Vorbereitung eines European Technology Assessment Networks (ETAN) und der dazugehörigen Infrastruktur (ETAI). Diese sollten begleitend zum spezifischen Programm "Sozio-ökonomische Schwerpunktforschung" (besser bekannt unter seinem englischen Akronym TSER - Targeted SocioEconomic Research) eingerichtet werden, das als Bestandteil des Vierten Rahmenprogramms erstmals ein eigenes sozialwissenschaftliches
Forschungsprogramm der EU darstellte. Einer der Schwerpunkte des TSER-Programmes ist denn auch Technology Assessment.

Während die Zuständigkeit für die Aktivitäten zur Vorbereitung des Aufbaus des Netzwerks und die inhaltliche Vorbereitung des TSER-Programms gegen Ende des Dritten Rahmenprogramms bei zwei unterschiedlichen Generaldirektionen (DG XII und XIII) lag, sollte mit Inkrafttreten des Vierten Rahmenprogramms allein die Generaldirektion XII (Forschung) sowohl für das TSER-Programm als auch für ETAN zuständig sein.

Die vorbereitenden Aktivitäten, die zum Teil unter dem Dach des VALUE Programms von DG XIII stattfanden, führten zu zahlreichen Kontakten innerhalb der europäischen TACommunity und gaben Anlaß zu optimistischen Erwartungen bezüglich der Ausbreitung der TA-Idee in Europa innerhalb eines geeigneten organisatorischen Rahmens.

\section{Die Rolle von Technology Assessment im Vierten Rahmenprogramm}

Die wissenschaftlich-technischen Ziele der Aktivitäten der EU im Bereich Forschung und technologische Entwicklung werden wie folgt beschrieben:

"Sie streben eine Verbesserung der Wettbewerbsfähigkeit der europäischen Industrie und der Lebensqualität an, und sie wurden konzipiert, um die wissenschaftlich-technischen Grundlagen zu schaffen, die zur Unterstützung einer nachhaltigen Entwicklung, des Umweltschutzes und weitere gemeinsame Politikbereiche benötigt werden" (CORDIS focus, Beilage, 20 Mai 1994:9, ÜdVerf.).

TA und ähnliche Aktivitäten waren nicht auf das TSER Programm beschränkt: "Neben der spezifischen sozio-ökonomischen Schwerpunktforschung (..) wird Forschung in den Geistes- und Sozialwissenschaften mit Forschung im Bereich Human- und Naturwissenschaften und Ingenieurwesen koordiniert, insbesondere im Hinblick auf die Erkundung des sozio-ökonomischen Umfeldes der geplanten Aktivitäten und ihrer möglichen Folgen" (ebd., S. 10).

Es gibt einen eigenen Abschnitt $\mathrm{zu}$ "Abschätzung wissenschaftlich-technischer Politikoptionen", der die Bedeutung der Forschung für Entscheidungsträger hervorhebt: "Das Ziel ist 
es, den an Forschung und Entwicklung beteiligten Akteuren, Entscheidungsträgern und Benutzern einen konsistenten Rahmen zur Bewertung der wissenschaftlich-technischen Optionen $\mathrm{zu}$ bieten, die mit Aktivitäten auf regionaler, nationaler und europäischer Ebene verbunden sind" (ebd., S. 21).

Erwähnt wird auch die Absicht, parlamentarische Einrichtungen, wie das Scientific and Technological Options Assessment Project (STOA) der Europäischen Kommission und die anderen Mitglieder des europäischen parlamentarischen TA-Netzwerks (EPTA), in diese Aktivitäten einzubeziehen. Ähnliches gilt für "wissenschaftliche Netzwerke zur Abschätzung von Wissenschafts- und Technologiepolitik auf regionaler, nationaler und europäischer Ebene" (ebd.).

Eine besondere Rolle war für das Institute for Prospective Technological Studies (IPTS, http://www.jrc.es) der Gemeinsamen Forschungsstelle (GFS) der Kommission vorgesehen. $\mathrm{Zu}$ den Aufgaben dieses Instituts sollte es gehören, "ein technologisches Observatorium einzurichten, das zur Sammlung und Analyse von Informationen $\mathrm{zu}$ wissenschaftlichen Ereignissen und technologischen Innovationen bei Berücksichtigung anderer relevanter internationaler Wissenschaftsorganisationen in diesem Bereich, sowie zur Durchführung von zukunftsgerichteten Studien und technologischen Bewertungen im wesentlichen auf Anforderung von Einrichtungen der Gemeinschaft dienen sollte" (ebd., S. 21).

Es bestand die Absicht, sämtliche Initiativen zu TA, die vor Inkrafttreten des Vierten Rahmenprogramms in die Wege geleitet wurden, unter dem Dach des TSER-Programms zu bündeln. Das Netzwerk ETAN sollte dabei als organisatorischer Rahmen für die Koordination, Sammlung und Verteilung von Information sowie zur Debatte dienen. Auch das vorgesehene Observatorium sollte eine Untergliederung von ETAN sein. Das ursprüngliche, im hohen Maße zentralisierte Konzept für das ETAN Netzwerk wurde auf einem Treffen hochrangiger Experten im Oktober 1994 vorgestellt und stieß auf heftige Kritik, unter anderem, weil die Entwicklung des Konzepts weitgehend abgekoppelt von der laufenden Diskussion innerhalb der TA-Community erfolgt war.

\section{TA in der Praxis des Vierten Rahmenpro- gramms}

Das Vierte Rahmenprogramm trat am 1. Januar 1995 in Kraft. Zum selben Termin traten Österreich, Finnland und Schweden der EU bei. Beide Ereignisse waren Anla $\beta$ für eine radikale Umverteilung von Zuständigkeiten innerhalb der Kommissionsdienststellen. So wurde ein neues Direktorat innerhalb von DG XII geschaffen, um das TSER-Programm und ETAN zu betreuen. Ein erster Aufruf zur Einreichung von Forschungsanträgen und Vorschlägen für "thematische Netze" im Rahmen von TSER wurde Mitte März 1995 veröffentlicht. Ebenfalls Mitte März 1995 gab es einen ersten Aufruf zur Bekundung von Interesse an der Mitgliedschaft von ETAN, dem mehrere Hundert Einrichtungen folgten.

Während das TSER-Programm zu einer Reihe von Projekten und thematischen Netzwerken, auch im Umfeld von TA, geführt hat, herrschte im Hinblick auf ETAN lange Zeit Funkstille. Unterdessen wurden mehrere unterschiedliche Konzepte für dieses Netzwerk entwickelt und meist schnell wieder verworfen. Der zweite Aufruf für Projektvorschläge im Rahmen des TSER-Programms wurde mehrmals verschoben, was insgesamt auf organisatorische Probleme im Bereich der zuständigen Direktion hindeutet. Schließlich wurden im September 1996 die Zuständigkeiten für TSER und ETAN auf zwei getrennte Direktionen aufgeteilt.

Parallel zu den TSER und ETAN-Aktivitäten nahm am 1. Januar 1995 das IPTS seine bereits beschriebenen Aufgaben in Angriff. Das Arbeitsprogramm des Instituts besteht aus drei Säulen, die sich aus dem Vierten Rahmenprogramm ergeben haben:

- Die Technikbeobachtung, d.h. die Beobachtung der wissenschaftlich-technischen Entwicklung mit dem Ziel des Aufzeigens "wissenschaftlicher Durchbrüche, von Tendenzen und Ereignissen, die zu technischen Innovationen führen könnten, und EU-Entscheidungsträger auf deren Implikationen und Folgen hinzuweisen, insbesondere falls Handeln auf europäischer Ebene erforderlich werden sollte" (IPTS Jahresbericht 1995:5). Zu diesem Zweck hat das IPTS das European Science and 
Technology Observatory (ESTO) gegründet, ein Netzwerk von Einrichtungen. Die für Forschung, Bildung und Weiterbildung zuständige Kommissarin, Mme. Edith Cresson, ermutigte das IPTS ein Mittel zu schaffen, das den Zweck der Verbreitung der Ergebnisse von Technikbeobachtungsaktivitäten unter Entscheidungsträgern innerhalb der EU haben soll. Dieses Ziel wird mit dem "IPTS Report" angestrebt, einer Zeitschrift, die zehn mal jährlich erscheint.

- Technologie-Beschäftigung-Wettbewerbsfähigkeit: Auf diesem Arbeitsgebiet will das IPTS zum Verständnis über die $\mathrm{Zu}$ sammenhänge zwischen Technologie, Beschäftigung und der Wettbewerbsfähigkeit Europas beitragen, und auch darüber, wie die Technologie neue Bedürfnisse, Märkte und Dienstleistungen erzeugt.

- Arbeit auf Anforderung: "Das Ziel dieser Art von Aktivitäten ist die Unterstützung der Entwicklung und/oder Umsetzung von EU Politik mit solider Grundlage in Wissenschaft und Technik und einer gemeinsamen Wissensbasis" (ebd., S. 6). Ihre Hauptauftraggeber sind die Dienststellen der Kommission und das Europäische Parlament, genauer die STOA-Einheit. Das IPTS führte 1995 ein erstes Projekt für STOA durch und ist offensichtlich bestrebt, zunehmend auch für das Europäische Parlament zu arbeiten.

Das IPTS Arbeitsprogramm sah die Schaffung des ESTO-Netzwerks als Untergliederung von ETAN vor. Der Kern von ESTO bestand ursprünglich aus zehn Einrichtungen, die vom IPTS vertraglich verpflichtet wurden, ESTO mit aufzubauen. Das vollständige Netzwerk bestand aus etwa 50 Einrichtungen, die vom IPTS oder den Mitgliedern des Kernnetzwerks vorgeschlagen wurden. Die ersten Monate der Existenz von ESTO dienten überwiegend dem Aufbau des "IPTS Reports", dessen Artikel in der Anfangszeit hauptsächlich von den IPTSMitarbeitern geschrieben und von den ESTOMitgliedern kommentiert wurden.

Gegen Ende dieser Gründungsphase wurde vom IPTS das Konzept des ESTO-Netzes revidiert. Eine begrenzte Ausschreibung fand statt, die an Netzwerke von Einrichtungen gerichtet war, die das IPTS beim Ausbau und Betrieb des ESTO-Netzwerks unterstützen sollten. Die dafür erforderlichen Leistungen und Artikel für den "IPTS Report" sollten honoriert werden. Mit diesem Konzept strebte das IPTS vermutlich an, wichtige nationale Akteure als "Knoten" zu verpflichten, um damit die "Netzwerke" dieser Knoten auch in ESTO-Aktivitäten einzubinden.

Der Vertrag ging an ein Konsortium von 14 Einrichtungen aus 11 europäischen Ländern, das von der Abteilung "Zukünftige Technologien" des VDI-Technologienzentrums Düsseldorf koordiniert wird. 1997 trat der Vertrag in Kraft. Aus Deutschland ist das ITAS, neben dem VDI-TZ, an der Kerngruppe von ESTO beteiligt.

Im ersten Jahr mit neuem Konzept wurden eine ganze Reihe kleiner Projekte durchgeführt, zahlreiche Beiträge für den "IPTS Report" geschrieben und eine erste Ausgabe eines jährlich vorgesehenen Berichts zur technisch-ökonomischen Entwicklung im Entwurf erstellt. Diese Arbeiten waren weitestgehend auf die Mitglieder des Konsortiums beschränkt. Das IPTS selbst führte in dieser Phase ein größeres Projekt mit Titel "Made in Europe" durch, das sich erkennbar am Vorbild des "Made in USA"Berichts orientierte.

Die Arbeiten des ESTO-Netzwerks waren 1997 unter anderem dadurch geprägt, daß das IPTS kommissarisch geleitet wurde und deshalb Unklarheit über die längerfristige Ausrichtung des Arbeitsprogrammes sowohl von ESTO als auch vom IPTS bestand. Der Amtsantritt des neuen Direktors des IPTS im Frühjahr 1998 wurde zum Anlaß genommen, das bisherige Konzept zu überdenken, und die Arbeiten des ESTO zukünftig enger mit dem Arbeitsprogramm des IPTS zu verzahnen. So gibt es wenige kleinere Projekte, die sich aber stark an den Bedürfnissen von Auftraggebern innerhalb der Einrichtungen der EU orientieren. Ein Großteil der Ressourcen von ESTO für 1998 und 1999 wurden nunmehr für das Nachfolgeprojekt von "Made in Europe" vorgesehen, das den Titel "Futures" trägt, und führende Experten in eine Abschätzung der Herausforderungen in den ersten Jahrzehnten des neuen Jahrtausends einbinden will. Dazu soll es zahlreiche Panels und Workshops geben. 
Kennzeichnend für die Aktivitäten von ESTO in 1998 ist das Bestreben, das Netzwerk über den Kern hinaus zu öffnen.

1997/98 wurde auch das Konzept von ETAN vollständig modifiziert. "Zweck des ETAN-Netzwerkes ist es, die Kommunikation und Diskussion zwischen Wissenschaftlern und politischen Entscheidungsträgern über zentrale Themen im Bereich der Wissenschaft und Technologie (W\&T-Politik) zu fördern" (zitiert nach ${ }^{2}$ ) (http://www.cordis.lu/etan/home.html).

ETAN beruft zu ausgewählten Fragestellungen Arbeitsgruppen von Experten. Diese Arbeitsgruppen werten vorhandene Forschungsergebnisse im Themenbereich für ETAN-Arbeitspapiere mit Problemdarstellungen und Entscheidungsoptionen aus. Die Fragestellungen, die von den Arbeitsgruppen besonders thematisiert werden, sind Gegenstände von Seminaren und Konferenzen, an denen Experten, Entscheidungsträger und sonstige Betroffene teilnehmen.

Die ersten drei Themen, die von solchen Expertengruppen aufgegriffen wurden, sind:

- Technologiepolitik im Kontext der Internationalisierung: Wie können Europas Wettbewerbsvorteile im Bereich Technik gestärkt werden?

- Die alternde Bevölkerung und die Technik: Herausforderungen und Chancen. Diese Arbeitsgruppe hat ihre Arbeit bereits abgeschlossen und einen Bericht vorgelegt. ${ }^{2}$

- Antworten der Forschungs- und Entwicklungspolitik auf die Herausforderungen des globalen Klimawandels. In dieser Arbeitsgruppe arbeitet das ITAS mit.

Im Rahmen von ETAN besteht schließlich die Möglichkeit, zum Bereich "Strategische Analysen $\mathrm{zu}$ spezifischen politischen Fragestellungen" der Aktionslinie "Unterstützung der Entwicklung von Wissenschafts- und Technologiepolitik in Europa" des vorgesehenen spezifischen Programms "Verbesserung des menschlichen Forschungspotentials und der sozio-ökonomischen Wissensbasis" mit Kommentaren und Anregungen beizutragen. Dies wird wohl der Hauptkristallisationspunkt von TA und verwandten Aktivitäten im Fünften Rahmenprogramm sein.

Nach einigen Verzögerungen sind Strukturen für TA-Aktivitäten auf EU-Ebene etabliert.
Es bleibt zu hoffen, daß sie reibungslos auch für das Fünfte Rahmenprogramm übernommen werden können.

\section{Anmerkungen}

1 ETAN-Working Papers: The Aging Population and Technology: Challenges and Opportunities. Europäische Kommission, DG XII-AS, Februar 1998

\section{EPTA - das Europäische Par- lamentarische TA-Netzwerk}

\author{
von Ingrid von Berg, ITAS
}

Nachdem in den USA bereits 1972 durch die Gründung des Office of Technology Assessment (OTA) beim U.S. Kongre $B$ Technikfolgenabschätzung auf parlamentarischer Ebene institutionalisiert wurde, entschieden sich europäische Länder erst sehr viel später und nach zum Teil sehr langen und kontroversen Diskussionen dafür, parlamentarische TA-Einrichtungen zu etablieren. Einige der Einrichtungen wurden zunächst auf befristete Zeit eingerichtet (wie auch das Büro für Technikfolgen-Abschätzung beim Deutschen Bundestag) und dann einer Evaluation unterzogen. Alle Institutionen haben sich jedoch inzwischen als fester Bestandteil der parlamentarischen Beratungs- und Entscheidungsprozesse etablieren können. 1990 wurde das European Parliamentary Technology Assessment Network gebildet (EPTA).

\section{Gründung und Mitgliedsorganisationen}

Im Jahre 1990 haben sich die Büros der damals sechs parlamentarischen Einrichtungen in Dänemark, Deutschland, Frankreich, Großbritannien, Niederlande und beim Europäischen Parlament unter der Schirmherrschaft des Präsidenten des Europäischen Parlaments, Enrique Baron Crespo, formell zum Europäischen Parlamentarischen TA-Netzwerk: European Parliamentary Technology Assessment Network (EPTA) zusammengeschlossen. Die Initiative hierzu ging von Lord Kennet, Vorsitzender des 